\title{
Local States in One-Dimensional Symmetrical Quantum Systems
}

\author{
Jürgen Brickmann
}

Physikalische Chemie I, Technische Hochschule Darmstadt

Z. Naturforsch. 34a, 1452-1457 (1979); received September 12, 1979

Local quantum states, which play an important role in quantum dynamical treatments, are expanded analytically with respect to a basis of eigen functions of a symmetrical Hamiltonian $\hat{\mathscr{H}}(x)=\hat{\mathscr{H}}(-x)$. Exact local states (ELS) in one-dimensional symmetrical quantum systems are therein defined as quantum states which are local eigenstates of the Hamiltonian $\mathscr{H}(x)$ on one half space $\mathbb{R}_{+}$or $\mathbb{R}_{-}$and are identically equal to zero on the other half space. Local properties like the projection operator on one half space can be given in terms of ELS-basis, but it is shown that the energy moments $\left\langle(\langle\hat{\mathscr{H}}\rangle-\hat{\mathscr{H}})^{k}\right\rangle$ with respect to the ELS do not converge. Consequently, if one uses the ELS as quasistationary initial states, as has been done recently by some authors [5], the lifetimes of these states cannot be estimated from time energy uncertainty relation using the second energy moment as an energy uncertainty measure. A harmonic oscillator system and a symmetrical double oscillator are treated as examples.

\section{Introduction}

It can be said that a lot of physical and chemical phenomena like the decay of nuclear and atomic states, diffusion in solids [1], reorientational motions in solids [2], and molecular rearrangements [3-5] can be described in terms of wave packets which are more or less localized on some spatial domain at an initial time. If the creation process of these initial states is well known then this is also true for the wave packet, at least in principle. Very often the information on such initial states is incomplete, i.e. one can only establish probabilities. In the latter case the initial situation can be described with the aid of the density matrix formalism. The simplest initial density matrix, for example, for a canonical ensemble, is a diagonal one with respect to a basis of exact local "eigenfunctions" (ELS) of the Hamiltonian $\hat{\mathscr{H}}$. These local eigenfunctions describe the possible quantum states of a particle which is not able to leave the localization domain. They may be defined as functions which are eigenfunctions of $\hat{\mathscr{H}}$ inside the domain and which are equal to zero on the outside. The situation here is somewhat similar to that recently discussed by Dollard [6], i.e. we are looking for eigenfunction $\psi \in L_{2}(R)$ of $\hat{\mathscr{H}}_{\mathrm{L}}$, with $\hat{\mathscr{H}}_{\mathrm{L}}=\hat{\mathscr{H}}+\mathscr{V}$ where $\mathscr{V}$ represents an infinite $\delta$-function potential located at the ends of the localization domain. Our aim is to expand the eigenfunction of $\hat{\mathscr{H}}_{\mathrm{L}}$ with respect to the eigen-

Reprint requests to Prof. Dr. J. Brickmann, Physikalische Chemie I, Technische Hochschule Darmstadt, Petersenstraße 20, D-6100 Darmstadt. functions of $\hat{\mathscr{H}}$ and hereby give meaning to the operator $\hat{\mathscr{H}}_{\mathrm{L}}$ on the Hilbert space $L^{2}(\mathbb{R})$. As a consequence of such expansion all operators which describe local properties on $\mathbb{R}_{+}$or $\mathbb{R}_{-}$can be given in terms of a basis of eigenstates of $\hat{\mathscr{H}}$.

\section{The Hamiltonian}

We would like to consider a quantum mechanical particle moving in a one-dimensional symmetrical potential which is bound from below.

The Hamiltonian of the system may be given as

$$
\hat{\mathscr{H}}(x)=-\frac{1}{2 m} \frac{\mathrm{d}^{2}}{\mathrm{~d} x^{2}}+\mathscr{V}(x)
$$

with $\mathscr{V}(x)=\mathscr{V}(-x)$. Consequently, the eigenfunctions of $\hat{\mathscr{H}}$ are either odd or even functions $u_{i}(x)$ and $g_{i}(x)$ respectively.

$$
\begin{aligned}
& \hat{\mathscr{H}} u_{i}(x)=E_{i}{ }^{u} u_{i}(x), \\
& \mathscr{H} g_{i}(x)=E_{i}{ }^{g} g_{i}(x) .
\end{aligned}
$$

One always has $E_{i}{ }^{u} \geqq E_{i}{ }^{g}$. For the following terms we introduce the short notation

$$
E_{i}{ }^{u}=E_{i}, \quad E_{i}{ }^{g}=E_{i}-\Delta E_{i} .
$$

Accordingly, from the symmetry of the system one obtains

$$
u_{i}(0)=0, \quad g_{i}{ }^{\prime}(0)=\mathrm{d} g_{i}(x) /\left.\mathrm{d} x\right|_{x=0}=0 .
$$

In many cases such as isomerization reaction, diffusion models, etc. one is interested in analysing the time evolution of localized wave packets. If $\varphi(x)$ is such a localized square integrable function 
with $\|\varphi(x)\|=1$ at $t=0$, the time evolution is easily calculated as follows

$$
\begin{aligned}
& \varphi(x, t)= \exp (-i \hat{\mathscr{H}} t) \varphi(x) \\
&=\sum_{j}\left\{\left\langle u_{j} \mid \varphi\right\rangle \exp \left(-i E_{j} t\right) u_{j}(x)\right. \\
&\left.\quad+\left\langle g_{j} \mid \varphi\right\rangle \exp \left(-i\left(E_{j}-\Delta E_{j}\right) t\right) g_{j}(x)\right\} .
\end{aligned}
$$

From this formula we can see that the time evolution of $\varphi(x)$ is completely determined from the energy eigenvalues of $\hat{H}$ and the expansion coefficients $\left\langle g_{i} \mid \varphi\right\rangle$ and $u_{i}|\varphi\rangle$.

\section{Expansion Coefficients of Exact Local States (ELS)}

In this paper we wish to report on our analyses with initial wave packets $l_{i}(x)$ and $r_{i}(x)$ which are initially strictly localized on one half space $\mathbb{R}_{+}$ or $\mathbb{R}_{-}$, and which are local eigenfunctions of the Hamiltonian $\hat{H}$ on $\mathbb{R}_{+}$or $\mathbb{R}_{-}$with boundary conditions $l_{i}(x)=0$ on $\mathbb{R}_{+}$and $r_{i}(x)=0$ on $\mathbb{R}_{-}$. These states represent a particle which is bounded on $\mathbb{R}_{+}$ of $\mathbb{R}_{-}$respectively, i.e. each localized state can be expanded with respect to a basis of the $l_{i}(x)$ or the $r_{i}(x)$.

Furthermore, it is to be noted that since the $u_{i}(x)$-states fulfill the boundary condition $u_{i}(x)=0$ for $x=0$, the local states can easily be obtained from these states.

$$
\begin{aligned}
& l_{i}(x)=\left\{\begin{array}{cl}
2^{1 / 2} u_{i}(x) & \text { for } \quad x \in \mathbb{R}_{-}, \\
0 & \text { else } ;
\end{array}\right. \\
& r_{i}(x)=\left\{\begin{array}{cl}
0 & \text { for } \quad x \in \mathbb{R}_{-}, \\
-2^{1 / 2} u_{i}(x) & \text { else. }
\end{array}\right.
\end{aligned}
$$

The expansion coefficients $\left\langle g_{j} \mid l_{i}\right\rangle,\left\langle u_{j} \mid l_{i}\right\rangle,\left\langle g_{j} \mid r_{i}\right\rangle$ and $\left\langle u_{j} \mid r_{i}\right\rangle$ can now be given in a closed form

$$
\begin{aligned}
\left\langle u_{j} \mid l_{i}\right\rangle= & 2^{-1 / 2} \delta_{j i}, \\
\left\langle u_{j} \mid r_{i}\right\rangle= & -2^{-1 / 2} \delta_{j i} .
\end{aligned}
$$

For $i=j$ this relation directly follows from the definition Eq. (6), for $i \neq j$ one can use the orthonormality relation $\left\langle u_{i} \mid u_{j}\right\rangle=\delta_{i j}$ and the fact that the integrand is an even function of the spatial coordinate, meaning that the integral has to be equal to zero on $\mathbb{R}_{+}$or $\mathbb{R}_{-}$if it vanishes over $R$.

In order to determine the coefficients $\left\langle g_{j} \mid l_{i}\right\rangle$ and $\left\langle g_{j} \mid r_{i}\right\rangle$ we first notice that

$$
\left\langle g_{j} \mid r_{i}\right\rangle=\left\langle g_{j} \mid l_{i}\right\rangle
$$

which can easily be seen from (6). Moreover, one has the following relations

$$
\begin{aligned}
\hat{\mathscr{H}} l_{i}(x)= & -\frac{1}{2 m} \frac{\mathrm{d}}{\mathrm{d} x}\left\{\begin{array}{cl}
2^{1 / 2} u_{i}{ }^{\prime}(x) & \text { for } x \in \mathbb{R}- \\
0 & \text { else }
\end{array}\right\} \\
& +\mathscr{V}(x) l_{i}(x) \\
= & E_{i} l_{i}(x)+\frac{1}{2 m} \frac{\mathrm{d}}{\mathrm{d} x} \Theta(x) \cdot 2^{1 / 2} u_{i}{ }^{\prime}(0) \\
= & E_{i} l_{i}(x)+\left(2^{-1 / 2} / m\right) u_{i}{ }^{\prime}(0) \delta(x)
\end{aligned}
$$

with the Heavyside function $\Theta(x)$ and the Dirac $\delta$-function

$\Theta\left(x-x_{0}\right)=\left\{\begin{array}{lll}0 & \text { for } & x<x_{0} \\ 1 & \text { for } & x \geqq x_{0}\end{array}\right\}=\int_{-\infty}^{x} \mathrm{~d} \xi \delta\left(\xi-x_{0}\right)$.

Multiplication of Eq. (9) from the left with $g_{j} *(x)$ and integrating over $\mathbb{R}$ gives us for the lhs.

$$
\left\langle g_{j}|\hat{\mathscr{H}}| l_{i}\right\rangle=\left(E_{j}-\Delta E_{j}\right)\left\langle g_{j} \mid l_{i}\right\rangle
$$

and from the rhs of Eq. (9) one obtains

$$
\begin{aligned}
E_{i}\left\langle g_{j} \mid l_{i}\right\rangle & +\left(2^{-1 / 2} / m\right) u_{i}{ }^{\prime}(0) \int_{-\infty}^{\infty} g_{j} *(x) \delta(x) \mathrm{d} x \\
& =E_{i}\left\langle g_{j} \mid l_{i}\right\rangle+\left(2^{-1 / 2} / m\right) u_{i}{ }^{\prime}(0) g_{j}{ }^{*}(0) .
\end{aligned}
$$

Finally, from Eqs. (9)-(11) one obtains

$$
\left\langle g_{j} \mid l_{i}\right\rangle=g_{j}{ }^{*}(0) u_{i}{ }^{\prime}(0)\left(2^{-1 / 2} / m\right)\left(E_{j}-E_{i}-\Delta E_{j}\right)^{-1} \text {. }
$$

\section{Expectation Values of Operators with Respect to Local States and Quasi-Local States}

The expansion coefficients $\left\langle g_{j} \mid l_{i}\right\rangle$ can now be used to obtain expectation values of some operators $\hat{\mathcal{O}}$ with regard to the exact local states (ELS)

$$
\begin{aligned}
\langle\hat{\mathcal{O}}\rangle_{i} & \equiv\left\langle l_{i}|\hat{\mathcal{O}}| l_{i}\right\rangle \\
& =\sum_{j}\left\langle l_{i}|\hat{\mathcal{O}}| g_{j}\right\rangle\left\langle g_{j} \mid l_{i}\right\rangle+2^{-1 / 2}\left\langle l_{i}|\hat{\mathcal{O}}| u_{i}\right\rangle
\end{aligned}
$$

where we used (7). The expansion relation (14) is only valid if the rhs. converges; this, however, is not true for all operators. For example, for $\hat{\mathcal{O}}=\hat{\mathscr{H}}^{k}$, with $k \geqq 2$, the rhs of (14) is a divergent series, as can be seen when using (9). This statement has several consequences for lifetime calculations when using the time energy uncertainty relation

$$
\tau_{i}>\left(\Delta E_{i}\right)^{-1} \quad(\hbar=1 \text {-units })
$$

and if one identifies $\Delta E_{i}$ with the second moment of Hamiltonian $\Delta E_{i}{ }^{2}=\left\langle\left(\hat{\mathscr{H}}-\langle\hat{\mathscr{H}}\rangle_{i}\right)^{2}\right\rangle_{i}$. Expan- 
sion of $l_{i}(x)$, in terms of a $L^{2}(\mathbb{R})$ basis, yields $\Delta E_{i}=\infty$ and in turn the trivial result $\tau_{i}>0$.

In many cases, especially in numerical applications, only finite sets $\left\{\left|u_{k}\right\rangle,\left|g_{k}\right\rangle \mid k=1-N\right\}$ of eigenfunctions of $\hat{\mathscr{H}}$ can be applied for the expansion of the local states

$$
\left|l_{i}^{N}\right\rangle=\sum_{j=1}^{N}\left\langle g_{j} \mid l_{i}\right\rangle\left|g_{j}\right\rangle+2^{-1 / 2}\left|u_{i}\right\rangle
$$

with $1 \leqq i \ll N$. We will call such states quasilocal states (QLS). For the choice of $\hat{\mathcal{O}}=\hat{\mathscr{I}}$ (identity operator), Eq. (14) can be used to calculate the extent of localization

$$
0<G_{i}(N) \leqq \sum_{j=1}^{N}\left|\left\langle l_{i} \mid g_{j}\right\rangle\right|^{2}<1 / 2
$$

with $G_{i}(N) \rightarrow 1 / 2$ if $N \rightarrow \infty$. For each $G_{i}$ with $0<G_{i}<1 / 2$, one can find a finite integer $N\left(G_{i}\right)$ to fulfill relation (16), i.e. we can localized all states to the same extent by using different values of $N$. Such states will be designed as $\left|l_{i}^{G}\right\rangle$ and the corresponding expectation values are

$\langle\hat{\mathcal{O}}\rangle_{i}{ }^{G} \equiv\left\langle l_{i}{ }^{G}|\hat{\mathcal{O}}| l_{i}{ }^{G}\right\rangle \mid\left\langle l_{i}{ }^{G} \mid l_{i}{ }^{G}\right\rangle$

$=\left(\sum_{j=1}^{N(G)}\left\langle l_{i}|\hat{\mathcal{O}}| g_{j}\right\rangle\left\langle g_{j} \mid l_{i}\right\rangle+2^{-1 / 2}\left\langle l_{i}|\hat{\mathcal{O}}| u_{i}\right\rangle\right) /(G+1 / 2)$.

Contrary to the ELS the first derivatives of the quasi-local states (QLS) at the symmetry center are no longer discontinuous. The transition near $x=0$ can be made arbitrarily smooth, and correspondingly, $\langle\hat{\mathcal{O}}\rangle^{G}$ always converges. However, the price for this convergence is the fact that the QLS are only approximately localized in the desired domain, i.e. the wave functions have a nonvanishing tail in the wrong region.

A typical example for quasi-local states are the simple superpositions $\left|u_{i}\right\rangle \pm\left|g_{i}\right\rangle$ which are commonly used in models for molecular rearrangement dynamics $[1,15,17]$. The extent of localization in the latter states sensitively depends on the special potential and the quantum number, so that these states cannot be generally used to discuss local properties.

\section{Localized States in a Harmonic Oscillator System}

The eigenstates of the harmonic oscillator form the basis of numerous theoretical and numerical treatments [7]. This fact is one of the reasons why we have calculated the expansion coefficients for local states in that system. The second one is because the coefficient $\left\langle g_{j} \mid l_{i}\right\rangle$ can be given analytically for this system.

The Hamiltonian of the harmonic oscillator may be written in generalized coordinates $(m=1, \hbar=1$, $\omega=1$ )

$$
\mathscr{H}(x)=\frac{1}{2}\left(-\frac{\mathrm{d}^{2}}{\mathrm{~d} x^{2}}+x^{2}\right)
$$

with normalized eigenfunctions

$$
\begin{aligned}
u_{i}(x)= & {\left[2^{2 i-1}(2 i-1) ! \pi^{1 / 2}\right]^{-1 / 2} } \\
& \cdot \exp \left(-x^{2} / 2\right) H_{2 i-1}(x), \\
g_{i}(x)= & {\left[2^{2 i-2}(2 i-2) ! \pi^{1 / 2}\right]^{-1 / 2} } \\
& \cdot \exp \left(-x^{2} / 2\right) H_{2 i-2}(x),
\end{aligned}
$$

and eigenvalues $(i=1,2,3, \ldots)$

$$
\begin{aligned}
& E_{i} u \equiv E_{i}=2 i-1, \\
& E_{i}^{g}=E_{i}-1=2 i-2,
\end{aligned}
$$

$H_{n}(x)$ being the Hermite polynomials.

By using the well known properties of the harmonic oscillator wave functions [8] one can easily show that

$$
\begin{aligned}
u_{i}{ }^{\prime}(x) & =\frac{\mathrm{d}}{\mathrm{d} x} u_{i}(x) \\
& =(i-1 / 2)^{1 / 2} g_{i}(x)-i^{1 / 2} g_{i+1}(x),
\end{aligned}
$$

i.e. the coefficients Eq. (13) can only be calculated from the even functions. Finally, with the property of the Hermiteans [9]

$$
H_{2 n}(0)=(-1)^{n}[(2 n) ! / n !]
$$

one finally obtains

$$
\begin{aligned}
g_{j}(0)= & (-1)^{j-1}[(2 j-2) !]^{1 / 2} \pi^{-1 / 4} \\
& \cdot\left[(j-1) ! 2^{j-1}\right]^{-1}, \\
u_{i}{ }^{\prime}(0)= & (-1)^{i-1} 2^{1 / 2}[(2 i-1) !]^{1 / 2} \pi^{-1 / 4} \\
& \cdot\left[(i-1) ! 2^{i-1}\right]^{-1},
\end{aligned}
$$

and from Eq. (13)

$$
\left\langle g_{j} \mid l_{i}\right\rangle \frac{(-1)^{i+j}[(2 j-2) !(2 i-1) !]^{1 / 2}}{\pi^{1 / 2}(j-1) !(i-1) ! 2^{i+j-2}[2(j-i)-1]} .
$$

Having this information on hand we can now try to calculate the energy moments. From Eq. (14) 
one obtains with Eq. (20) and (25) formally

$$
\begin{aligned}
\left\langle\hat{\mathscr{H}}^{k}\right\rangle_{i}= & \sum_{j}\left(E_{j}-\Delta E_{j}\right)^{k}\left|\left\langle l_{i} \mid g_{j}\right\rangle\right|^{2}+\frac{1}{2} E_{i}{ }^{k} \\
= & \frac{1}{2}(2 i-1)^{k}+\frac{(2 i-1) !}{\pi\left[(i-1) ! 2^{i-1}\right]^{2}} \\
& \cdot \sum_{j} \frac{(2 j-2)^{k}(2 j-2) !}{[2(j-i)-1]^{2}[(j-1) !]^{2} 2^{2 j-2}} .
\end{aligned}
$$

For large values of $j$, the elements of the series in Eq. (26) follow the expression $(2 / \pi)^{1 / 2}(2 j)^{k-5 / 2}$ which can be easily shown with the aid of Stirlings formula for the large integers $n, n ! \approx n^{n} e^{-n}(2 \pi n)^{1 / 2}$. Therefore, this series is only convergent for $k=0$ ( $\hat{\mathscr{H}}_{0}=\hat{\mathscr{I}}$, identity operation) and $k=1$ which leads to the identity

$$
\begin{aligned}
\langle\hat{\mathscr{H}}\rangle_{i}=E_{i} & =2 i-1=\frac{(2 i-1) !}{\pi\left[(i-1) ! 2^{i-1}\right]^{2}} \\
& \cdot \sum_{j} \frac{(2 j-2)(2 j-2) !}{[2(j-i)-1]^{2}[(j-1) !]^{2} 2^{2 j-1}} .
\end{aligned}
$$

For the quasi-local states, the corresponding expectation values $\left\langle\mathscr{H}^{k}\right\rangle_{i}{ }^{G}$ can be calculated for all $k$, but it is to be seen from Table 1 that $\left\langle\mathscr{H}^{0}\right\rangle_{i}{ }^{G}$ and $\left\langle\mathscr{H}^{1}\right\rangle_{i}{ }^{G}$ converge towards the exact values with increasing localization parameter $G$ while

\begin{tabular}{|c|c|c|c|c|c|}
\hline$i$ & $E_{i}$ & ${ }_{10} \log (1 / 2-G)$ & $N_{i}(G)$ & $E_{i}-E_{i}{ }^{G}$ & $\Delta \mathscr{H}_{i}{ }^{G}$ \\
\hline \multirow[t]{3}{*}{1} & 1 & -2 & 3 & .122 & .77 \\
\hline & & -3 & 11 & .056 & 1.09 \\
\hline & & -4 & 46 & .027 & 1.56 \\
\hline \multirow[t]{3}{*}{2} & 3 & -2 & 5 & .145 & 1.08 \\
\hline & & -3 & 15 & .073 & 1.44 \\
\hline & & -4 & 61 & .035 & 2.05 \\
\hline \multirow[t]{3}{*}{3} & 5 & -2 & 6 & .175 & 1.27 \\
\hline & & -3 & 18 & .085 & 1.68 \\
\hline & & -4 & 71 & .041 & 2.38 \\
\hline \multirow[t]{3}{*}{4} & 7 & -2 & 7 & .199 & 1.42 \\
\hline & & -3 & 20 & .095 & 1.87 \\
\hline & & -4 & 79 & .045 & 2.64 \\
\hline \multirow[t]{3}{*}{5} & 9 & -2 & 9 & .194 & 1.62 \\
\hline & & -3 & 22 & .103 & 2.03 \\
\hline & & -4 & 86 & .049 & 2.86 \\
\hline \multirow[t]{3}{*}{6} & 11 & -2 & 10 & .209 & 1.74 \\
\hline & & -3 & 24 & .110 & 2.18 \\
\hline & & -4 & 92 & .052 & 3.05 \\
\hline \multirow[t]{3}{*}{7} & 13 & -2 & 11 & .222 & 1.86 \\
\hline & & -3 & 26 & .115 & 2.31 \\
\hline & & -4 & 98 & .055 & 3.23 \\
\hline
\end{tabular}
$\left(\Delta \mathscr{H}_{i}{ }^{G}\right)^{2}=\left\langle\left(\mathscr{H}-\left\langle\mathscr{H}_{i}\right\rangle^{G}\right)^{2}\right\rangle_{i}{ }^{G}$ does not. The latter value increases by about half an oscillator quantum

Table 1. Localized states in a harmonic oscillator system (Energies in $\hbar \omega$-units). when the localization error decreases by a factor of ten, i.e. when $G$ changes from 0.49 to 0.499 .

Generally speaking, the divergence of the width $\Delta \mathscr{H}_{i}$ in the harmonic oscillator system has no severe consequences for practical applications since the localized states in this system will only play the role of basis states and not that of physically reasonable initial states. The situation changes, however, on the double oscillator system, which will be discussed in detail in the next chapter.

\section{Local States in a Double Oscillator System}

The simplest case where the local states may be physically acceptable initial states is the double oscillator system $[1-5]$. In this model the particle moves in a one-dimensional symmetrical double well potential. A lot of work has been done during the last few years to calculate the eigenstates and eigenvalues for different double oscillator models $[10-13]$ but the results only differ quantitatively. The time dependence of different types of initial states in such systems has also been extensively studied $[1,3-5,14-18]$. In most papers $[1,15,17]$ superpositions of only two eigenstates $u_{i}(x)$ (odd function) and $g_{i}(x)$ (even) were used as initial states. The time dependence of such state is then only a function of the splitting $\Delta E_{i}$; we designed this scheme as splitting approximation. The validity of it can be measured by the expectation value $W_{i}$ of the projection operator

$$
\begin{aligned}
& \hat{\mathscr{P}}_{i}=\left|u_{i}\right\rangle\left\langle u_{i}|+| g_{i}\right\rangle\left\langle g_{i}\right|, \\
& W_{i}=\left\langle l_{i}\left|\hat{\mathscr{P}}_{i}\right| l_{i}\right\rangle=\left|\left\langle l_{i} \mid g_{i}\right\rangle\right|^{2}+1 / 2 .
\end{aligned}
$$

Other authors [16] introduced harmonic oscillator functions as initial wave packets where the oscillator frequency was obtained from the curvature of the potential function at the minima. In a former paper [3] we used approximate localized eigenfunction, whereby the localization was obtained numerically by adding a step function $A \Theta(x)$ with $A \gg E_{i}$ to the potential energy and solving the resulting Schrödinger equation. In very recent publications of Cribb et al. [5] also quasi-localized initial state were used and the lifetime were obtained from the uncertainty $\left(\Delta \mathscr{H}_{i}{ }^{G}\right)^{2}=\left\langle\left(\mathscr{H}-\left\langle\mathscr{H}_{i}\right\rangle_{i}\right)^{2}\right\rangle_{i}{ }^{G}$, even though the extent of localization was not yet explicitly determined.

In this work we present a double oscillator model with potential energy $V(x)=0.005 x_{4}-0.25 x$ (same 
units as in the oscillator system). The potential minima are located at $x_{0}= \pm 5$, the curvature at this point is equal to that of the oscillator discussed above $V^{\prime \prime}\left(x_{0}\right)=1$, the barrier separating the two wells being $V_{0}=3.125$. The Schrödinger equation was solved numerically by linear variational calculations using 140 oscillator eigenfunctions (with $\omega=1$ ) as a basis. Three pairs of eigenvalues are below the barriers, the fourth pair is approximately equal to $V_{0}$, while all other eigenvalues are larger than $V_{0}$. For further information, some numerical results are listed in Table 2 . Concerning the lowestwo energy pairs $(i=1,2)$, the localized state can be well approximated in the splitting approximation as long as the difference of the localization parameter $G$ from $1 / 2$ is not less than $10^{-7}$.

In this case, the energy uncertainty $\Delta \mathscr{H}_{i}{ }^{G}$ is equal to $\Delta E_{i} / 2$, however. This is no longer true for $G \rightarrow 1 / 2$, as is to be seen from the third pair of eigenstates. Although the splitting approximation seems to be sufficiently fulfilled $\left(W_{3}=0.998\right)$, the uncertainty increases drastically with increasing localization, $\Delta \mathscr{H}_{3}{ }^{G}=0.0026 \approx \Delta E_{3} / 2$ for $G=0.49$ and $\triangle \mathscr{H}_{3}{ }^{G}=0.048$ for $G=0.4999$. On the other hand, for low localization accuracy, the uncertainty $\Delta \mathscr{H}_{3}{ }^{G}$ is approximately equal to the splitting $\Delta E_{i} / \mathbf{2}$.

\section{Concluding Comments}

It has been clearly shown in this paper that exact localized states (ELS) of a symmetrical onedimensional quantum system can be expanded in an infinite series with respect to the eigenfunctions of a given symmetrical Hamiltonian $\hat{\mathscr{H}}(x)=\hat{\mathscr{H}}(-x)$ and that the expansion coefficients can be given in closed analytic form if one knows the eigenvalues of $\hat{\mathscr{H}}$, the even eigenfunctions at the symmetry center and the first derivatives of the odd eigenfunctions at that point. The ELS are therein defined as continuous functions on $R$ which are eigenfunctions of $\hat{\mathscr{H}}$ in one half space and which are identically equal to zero in the other. This definition is equivalent to that where the ELS are linear combinations $2^{1 / 2}\left(\left|g_{i}^{\infty}\right\rangle \pm\left|u_{i}^{\infty}\right\rangle\right)$ with the eigenstates $\left|g_{i}^{\infty}\right\rangle$ and $\left|u_{i}^{\infty}\right\rangle$ of a Hamiltonian $\hat{\mathscr{H}}^{\infty}$ which is identically equal to $\hat{\mathscr{H}}(x)$ for all $x \neq 0$ and has an infinite delta function barrier at the symmetry center $V^{\infty}(x)=\lim _{A \rightarrow \infty} A \delta(x)[6]$. As a ceonsequence of the definition of the ELS the first derivative of those functions at $x=0$ is not continuous. This fact leads to poor convergence and even divergence if one expands expectation values of certain Hermitean operators with respect to ELS. Especially the

Table 2. Localized states in a symmetrical double oscillator system with Hamiltonian

$$
\hat{\mathscr{H}}=\hbar \omega\left(-\frac{1}{2} \mathrm{~d}^{2} / \mathrm{d} x^{2}+0.005 x^{4}-0.25 x^{2}\right)
$$

(Energies in $\hbar \omega$-units).

\begin{tabular}{|c|c|c|c|c|c|c|c|}
\hline$i$ & $E_{i}$ & $1-W_{i}$ & $I_{\mathrm{loc}} *$ & $N_{i}(G)$ & $E_{i}-E_{i}^{G}$ & $\Delta H_{i}{ }^{G}$ & $\Delta E_{i}$ \\
\hline 1 & -2.6355019 & $7 \cdot 10^{-8}$ & -7 & 1 & $3 \cdot 10^{-7}$ & $3.06 \cdot 10^{-7}$ & $6.12 \cdot 10^{-7}$ \\
\hline 2 & -1.703068 & $1 \cdot 10^{-5}$ & -5 & 2 & $4 \cdot 10^{-5}$ & $4.65 \cdot 10^{-5}$ & $9.26 \cdot 10^{-5}$ \\
\hline 3 & -.85435 & $2 \cdot 10^{-3}$ & $\begin{array}{l}-2 \\
-3 \\
-4\end{array}$ & $\begin{array}{l}3 \\
4 \\
7\end{array}$ & $\begin{array}{l}.0035 \\
.0020 \\
.0008\end{array}$ & $\begin{array}{l}.0026 \\
.0189 \\
.0483\end{array}$ & .0051 \\
\hline 4 & -.0943 & 0.06 & $\begin{array}{l}-2 \\
-3 \\
-4\end{array}$ & $\begin{array}{r}5 \\
8 \\
16\end{array}$ & $\begin{array}{l}.020 \\
.009 \\
.004\end{array}$ & $\begin{array}{l}.11 \\
.18 \\
.26\end{array}$ & .96 \\
\hline 5 & .666 & 0.19 & $\begin{array}{l}-2 \\
-3 \\
-4\end{array}$ & $\begin{array}{r}7 \\
12 \\
27\end{array}$ & $\begin{array}{l}.043 \\
.021 \\
.010\end{array}$ & $\begin{array}{l}.31 \\
.43 \\
.61\end{array}$ & $\cdot 33$ \\
\hline 6 & 1.537 & 0.26 & $\begin{array}{l}-2 \\
-3 \\
-4\end{array}$ & $\begin{array}{r}9 \\
15 \\
36\end{array}$ & $\begin{array}{l}.061 \\
.033 \\
.016\end{array}$ & $\begin{array}{l}.50 \\
.65 \\
.92\end{array}$ & .45 \\
\hline 7 & 2.529 & 0.27 & $\begin{array}{l}-2 \\
-3 \\
-4\end{array}$ & $\begin{array}{l}10 \\
17 \\
41\end{array}$ & $\begin{array}{l}.082 \\
.043 \\
.020\end{array}$ & $\begin{array}{r}.64 \\
.83 \\
1.19\end{array}$ & .51 \\
\hline
\end{tabular}

$* I_{\text {loc }}={ }_{10} \log (1 / 2-G)$. 
energy moments $\left\langle(\langle\hat{\mathscr{H}}\rangle-\hat{\mathscr{H}})^{k}\right\rangle$ diverge. Consequently, if one uses the ELS as quasistationary initial states [5], the lifetime of these states cannot be estimated from time energy uncertainty relation using the second energy moment as an energy uncertainty measure. One can avoid the discontinuity of the first derivatives of the ELS at $x=0$ by introducing quasi-localized states (QLS). These functions can be arbitrarily localized and made arbitrarily smooth near $x=0$ but exspectation values like the energy moments $\left\langle(\langle\hat{\mathscr{H}}\rangle-\hat{\mathscr{H}})^{k}\right\rangle$ for $k \geqq \mathbf{2}$ will become drastically dependent on the quasi-localization i.e. dependent also on the smoothness of the QLS near the symmetry center. Consequently, one has to be very

[1] J. A. Sussmann, Ann. Phys. Paris 6, 135 (1971).

[2] V. Narayanamurti and R. O. Pohl, Rev. Mod. Phys. 42, 201 (1970).

[3] J. Brickmann and H. Zimmermann, J. Chem. Phys. 50, 1608 (1969).

[4] J. Brickmann, Z. Naturforsch. 28a, 1759 (1973).

[5] P. H. Cribb, S. Nordholm, and N. S. Hush, Chem. Phys. 29, 31 (1978).

[6] J. D. Dollard, J. Math. Phys. 19, 806 (1978).

[7] See Ref. [3] and references given therein.

[8] See for example E. E. Anderson: Modern Physics and Quantum Mechanics, Saunders Co., Philadelphia 1971, pp. $176 \mathrm{ff}$.

[9] W. Magnus, F. Oberhettinger, and R. P. Soni, Formulas and Theorems for the Special Functions of Mathematical Physics, p. 250. Springer-Verlag, Berlin 1966. careful performing approximate calculations of quantities like lifetimes from energy moments with respect to quasi-localized states (QLS). This point will be discussed in a forthcoming paper [18].

\section{Acknowledgement}

I like to thank Susan Kyncl for carefully reading the manuscript and Dr. Michael Gerloff for performing the computational work. Financial support of the Deutsche Forschungsgemeinschaft, Bonn-Bad Godesberg and the Fonds der Chemischen Industrie, Frankfurt is also acknowledged. The numerical calculations were carried out with a TR-440-Computer of the computational center, University Konstanz.

[10] R. L. Samorjai and D. F. Hornig, J. Chem. Phys. 36, 1980 (1962).

[11] J. Brickmann, Z. Naturforsch. 30a, 1730 (1975) and references cited therein.

[12] P. Holzer, U. Mosel, and W. Greiner, Nucl. Phys. A 138, 241 (1969); J. Maruhn and W. Greiner, Z. Physik 251, 431 (1972).

[13] L. Wolniewicz and T. Orlikowski, J. Comp. Phys. 27, 169 (1978).

[14] J. H. Weiner, J. Chem. Phys. 68, 2492 (1978).

[15] P. Russegger and J. Brickmann, J. Chem. Phys. 62, 1086 (1975); J. Chem. Phys. 66, 1 (1977).

[16] M. C. Flanigan and J. R. de la Vega, J. Chem. Phys. 61, 1882 (1974).

[17] J. Brickmann, Ber. Bunsenges. Phys. Chem. 74, 331 (1970); 75, 747 (1971).

[18] J. Brickmann, will be published. 